MATHEMATICS OF COMPUTATION

Volume 66, Number 217, January 1997, Pages 207-213

S 0025-5718(97)00803-X

\title{
ITERATED SOLUTIONS OF LINEAR OPERATOR EQUATIONS WITH THE TAU METHOD
}

\author{
M. K. EL-DAOU AND H. G. KHAJAH
}

\begin{abstract}
The Tau Method produces polynomial approximations of solutions of differential equations. The purpose of this paper is (i) to extend the recursive formulation of this method to general linear operator equations defined in a separable Hilbert space, and (ii) to develop an iterative refinement procedure which improves on the accuracy of Tau approximations. Applications to Fredholm integral equations demonstrate the effectiveness of this technique.
\end{abstract}

\section{Canonical polynomials of linear operators}

Let $X$ be a separable Hilbert space having a basis $\mathbf{x}:=\left\{x_{0}, x_{1}, x_{2}, \ldots\right\}$, and let $\mathcal{A}(X)$ denote the space of all linear operators on $X$. Then, for $A \in \mathcal{A}(X)$ and for all $i \in \mathbf{N}, A x_{i}$ is a linear combination of the basis elements:

$$
A x_{i}=\sum_{j} a_{i j} x_{j}, \quad a_{i j} \in \mathbf{C} .
$$

For $A \in \mathcal{A}(X)$ and $x, y \in X$, we define the equivalence relation

$$
x \equiv_{A} y \quad \text { iff } \quad x-y \in \operatorname{ker} A .
$$

An operator $A$ is said to be banded-from-above if there exists an integer $k \in \mathbf{N}$ which satisfies the property $P(k)$ defined as:

$$
a_{i j}=0 \quad \forall i, j \geq 0 \text { with } j-i \geq k+1 .
$$

Let $\mathcal{A}_{b}(X) \subseteq \mathcal{A}(X)$ denote the subspace of all banded-from-above operators on $X$, and define an integer-valued function on the elements of $\mathcal{A}_{b}(X)$ by

$$
h(A):=\min \{k \in \mathbf{N}: P(k)\}
$$

which, following Ortiz [3, 4], is called the height of $A$. Then, if $A \in \mathcal{A}_{b}$ is of height $h(A)$ equation (1) becomes ${ }^{1}$

$$
A x_{i}=\sum_{j=0}^{h+i} a_{i j} x_{j}, \quad i \geq 0 .
$$

A polynomial of degree $n$ in $X$ is any finite linear combination of the form

$$
p_{n}=\lambda_{0} x_{0}+\lambda_{1} x_{1}+\cdots+\lambda_{n} x_{n}, \quad \lambda_{i} \in \mathbf{C}, \lambda_{n} \neq 0 .
$$

Received by the editor July 27, 1995.

1991 Mathematics Subject Classification. Primary 41A10, 41A65; Secondary 45B05, 47A50.

Key words and phrases. Tau Method, polynomial approximation, linear operator equations.

${ }^{1}$ When dealing with a particular operator $A$ we write $h$ instead of $h(A)$.

(C) 1997 American Mathematical Society 
Given a linear operator $A$ and an integer $n \geq 0$, we define an element $Q_{n} \in X$ in such a way that $A Q_{n}=x_{n}$; this $Q_{n}$ is called the $n$-th canonical polynomial associated with $A$. The following proposition provides explicit expressions for canonical polynomials of orders $\geq h$ :

Proposition 1. Let $A \in \mathcal{A}_{b}(X)$ be an operator of height $h$ and, with reference to (1), assume that $a_{i j}=1$ for all $i \geq 0$ and $j=h+i$. Then, the sequence of canonical polynomials $\left\{Q_{k}: k \geq h\right\}$ associated with $A$ is determined by the recursive formulae

$$
Q_{h+k} \equiv_{A} x_{k}-\sum_{i=0}^{h+k-1} a_{k, i} Q_{i}, \quad k \geq 0,
$$

where $Q_{h} \equiv_{A} x_{0}$.

Proof. We use the definition of $Q_{n}$ to write $x_{n}$ as $A Q_{n}$ and employ the linearity of $A$. For $k \geq 0$ we have

$$
\begin{aligned}
A x_{k} & =x_{h+k}+\sum_{i=0}^{h+k-1} a_{k i} x_{i} \\
& =A Q_{h+k}+\sum_{i=0}^{h+k-1} a_{k i} A Q_{i} \\
& =A\left[Q_{h+k}+\sum_{i=0}^{h+k-1} a_{k i} Q_{i}\right] .
\end{aligned}
$$

Therefore,

$$
Q_{h+k} \equiv_{A} x_{k}-\sum_{i=0}^{h+k-1} a_{k i} Q_{i}
$$

with $Q_{h} \equiv_{A} x_{0}$.

Note that in the above proposition, if $h>0$, the canonical polynomials $\left\{Q_{n}\right.$ : $n<h\}$ remain undefined. Furthermore, if $A$ is invertible, i.e. $\operatorname{ker} A=\mathbf{0}$, then we have equalities in place of equivalences modulo $\operatorname{ker} A$.

Corollary 2. Under the assumptions of Proposition 1, if $A$ is invertible, then the $n$-th canonical polynomial $Q_{n}$ is of degree $n-h$, where $n \geq h$.

\section{Approximation of linear operators with the Tau Method}

Let $A$ be an invertible linear operator satisfying the conditions of Proposition 1 with $h(A)=h \geq 0$. Consider the following operator equation with its linear auxiliary conditions (if any):

$$
\begin{aligned}
A u & =f, \\
B_{\mu} u & =g_{\mu}, \quad \mu=1,2, \ldots, m,
\end{aligned}
$$

where $g_{\mu} \in \mathbf{C}$ and $u \in X$ is the exact solution. The purpose of this section is to explain how the recursive formulation of the Tau Method, developed by Ortiz in $[3,4]$, can be applied to problem (2) in order to derive polynomial approximations of $u$. To this end, we proceed as follows: Assume $f$ is of the form

$$
f=\sum_{i=0}^{\alpha} f_{i} x_{i} \in X
$$


Then for some fixed $n \geq h+m$ we associate with (2) the following problem whose exact solution is denoted by $u_{n}$

$$
\begin{aligned}
A u_{n} & =f+\sum_{i=0}^{h+m-1} \tau_{n, i} x_{n-i}, \\
B_{\mu} u_{n} & =g_{\mu},
\end{aligned}
$$

where $\left\{\tau_{n, i}: i=0,1, \ldots, h+m-1\right\}$ are unknown parameters to be fixed with $n$. Taking $\left\{Q_{i}\right\}$ to be the set of canonical polynomials of $A$, we set

$$
U_{n}=\sum_{i=0}^{\alpha} f_{i} Q_{i}+\sum_{i=0}^{h+m-1} \tau_{n, i} Q_{n-i},
$$

and find that $A\left(u_{n}-U_{n}\right)=0$ by Proposition 1 ; since $A$ is invertible, it follows that $u_{n}=U_{n}$. It is not difficult to determine the Tau parameters: Since the canonical polynomials $\left\{Q_{i}: 0 \leq i<h\right\}$ are undefined, as mentioned earlier, we equate their coefficients in $U_{n}$ to zero and form a system of $h$ linear algebraic equations which, when added to the $m$ auxiliary conditions, will result in $h+m$ linear equations with an equal number of unknown $\tau$ 's.

The polynomial $u_{n}$ will be called the $n$-th Tau Method approximation to $u$ and, by Corollary 2 , its degree is $n$. The $n$-th Tau error is the difference $e_{n}:=u-u_{n}$, and the term $\sum \tau_{n, i} x_{n-i}$ in (3), denoted by $\rho_{n}$, is called the Tau perturbation term. The next result assures the convegence of the sequence $\left\{e_{n}: n \geq h+m\right\}$ to zero.

Theorem 3. If $X$ is a separable Hilbert space equipped with the inner product $\langle\cdot, \cdot\rangle$ and if $A \in \mathcal{A}_{b}(X)$ is invertible, then for fixed $h \geq 0$ and $m \geq 0$ in equations (2) and (3), we have

i. $\lim _{n \rightarrow \infty}\left|\tau_{n, i}\right|=0$ for $i=0,1, \ldots, h+m-1$.

ii. $\lim _{n \rightarrow \infty}\left\|e_{n}\right\|=0$.

Proof. Let $\left\{x_{i}: i \geq 0\right\}$ be a maximal orthonormal basis for $X$. Subtracting (3) from (2) we obtain the error equation

$$
A e_{n}=-\sum_{i=0}^{h+m-1} \tau_{n, i} x_{n-i}=-\rho_{n} .
$$

Since $A$ is invertible, it is bounded and so is its inverse $A^{-1}$. It follows then that $\left\|e_{n}\right\| \leq\left\|A^{-1}\right\| \cdot\left\|\rho_{n}\right\|$. Let $X_{n}=\operatorname{span}\left\{x_{0}, x_{1}, \ldots, x_{n-h-m}\right\}$ and take $X_{n}^{\perp}$ to be its orthogonal complement. Then, for each $j=0,1, \ldots, n-h-m$, we have $\left\langle\rho_{n}, x_{j}\right\rangle=0$ since $n \geq h+m$; thus $\rho_{n} \in X_{n}^{\perp}$. Since $\left\{X_{n}^{\perp}: n \geq h+m\right\}$ forms a decreasing sequence of closed linear subspaces of $X$ satisfying

$$
\lim _{n \rightarrow \infty} \operatorname{diam}\left(X_{n}^{\perp}\right)=0
$$

where $\operatorname{diam}(Y)=\sup \{\|a-b\|: a, b \in Y\}$ — see the addendum and [1, 2]—it follows from Cantor's theorem that

$$
\bigcap_{k=N}^{\infty} X_{k}^{\perp}=\{\mathbf{0}\}
$$

where $N=h+m$. But since

$$
\rho_{n} \in \bigcap_{k=N}^{n} X_{k}^{\perp},
$$


taking the limit as $n$ tends to infinity yields

$$
\lim _{n \rightarrow \infty} \rho_{n} \in \bigcap_{k=N}^{\infty} X_{k}^{\perp}=\{\mathbf{0}\},
$$

from which it follows that

$$
\lim _{n \rightarrow \infty}\left\|\rho_{n}\right\|=0 .
$$

Furthermore, from Parseval's identity we see that

$$
\left\|\rho_{n}\right\|^{2}=\sum_{i=0}^{h+m-1}\left|\tau_{n, i}\right|^{2}
$$

Combining (6) and (7) we get (i)

$$
\lim _{n \rightarrow \infty}\left|\tau_{n, i}\right|=0, \quad 0 \leq i \leq h+m-1,
$$

and (ii)

$$
\left\|e_{n}\right\| \leq\left\|A^{-1}\right\| \cdot\left\|\rho_{n}\right\| \rightarrow 0
$$

\section{Iterated TAu Method solutions}

The idea here is to start with the approximate solution $u_{n}$ of (2) and generate more accurate approximations via an iterative process. We may assume that the operator $A$ is of the form $A:=T-\lambda$ where $T \in \mathcal{A}_{b}(X)$ and $\lambda \in \mathbf{C}$. Equation (3) becomes

$$
T u_{n}-\lambda u_{n}=f+\rho_{n} .
$$

For $k \geq 0$, we define the $k$-th iterated Tau solution $\hat{u}_{n, k}$ associated with $u_{n}$ as follows

$$
\begin{aligned}
& \hat{u}_{n, 0}=u_{n}, \\
& \hat{u}_{n, k}=\lambda^{-1}\left(T \hat{u}_{n, k-1}-f\right), \quad k \geq 1 .
\end{aligned}
$$

Let the error $e_{n, k}=u-\hat{u}_{n, k}$. Then, for $k \geq 1$, we have

$$
\begin{aligned}
e_{n, k} & =\lambda^{-1}(T u-f)-\lambda^{-1}\left(T \hat{u}_{n, k-1}-f\right) \\
& =\lambda^{-1}\left(T u-T \hat{u}_{n, k-1}\right) \\
& =\lambda^{-k} T^{k}\left(u-u_{n}\right) \\
& =\lambda^{-k} T^{k} e_{n}
\end{aligned}
$$

and hence

$$
\left\|e_{n, k}\right\| \leq|\lambda|^{-k}\|T\|^{k} \cdot\left\|e_{n}\right\| .
$$

Since $e_{n}$ is independent of $k$, the right-hand side of this inequality will tend to zero as $k \rightarrow \infty$ if we assume $\|T\|<|\lambda|$. Thus, we have the main result of this paper:

Theorem 4. If $A=T-\lambda$ is invertible and if $\|T\|<|\lambda|$, then, for a fixed $n$, the following assertions hold:

i. $\left\|e_{n, k}\right\| \leq|\lambda|^{-k}\|T\|^{k} \cdot\left\|e_{n}\right\|, k \geq 0$,

ii. $\lim _{k \rightarrow \infty}\left\|e_{n, k}\right\|=0$. 
Note that from equations (8) and (9) we obtain the following procedure for computing $\hat{u}_{n, k}$ :

$$
\begin{aligned}
& \hat{u}_{n, 0}=u_{n}, \\
& \hat{u}_{n, k}=\hat{u}_{n, k-1}+\lambda^{-k} T^{k-1} \rho_{n}, \quad k \geq 1 .
\end{aligned}
$$

\section{NumericAL EXAMPLES}

We show, through the following two examples, how to improve the accuracy of Tau approximations using the procedure described above.

Example A. Consider the integral equation

$$
(F u)(t):=\int_{0}^{1} K(t, s) u(s) d s-\lambda u(t)=\phi(t), \quad t \in[0,1],
$$

where $\phi(t)$ is known. This defines an inhomogeneous Fredholm equation of the second kind with symmetric kernel

$$
K(t, s)= \begin{cases}-t(1-s) & \text { if } s \geq t, \\ -s(1-t) & \text { if } s \leq t .\end{cases}
$$

Taking the shifted Chebyshev polynomials $T_{k}^{*}(t)$ as basis elements, we approximate $u(t)$ in the subspace $X_{n}=\operatorname{span}\left\{T_{0}^{*}, T_{1}^{*}, \ldots, T_{n}^{*}\right\}$. We follow the recursive formulation of the Tau Method described in Ortiz $[3,4]$ for linear differential operators: Define $Q_{n}(t)$ so that

is satisfied. This leads to

$$
\left(F Q_{n}\right)(t)=T_{n}^{*}(t)
$$

$$
\begin{aligned}
& Q_{2}=16\left[T_{0}^{*}+\left(\frac{1}{16}+\lambda\right) Q_{0}\right], \\
& Q_{3}=96\left[T_{1}^{*}+\left(\frac{1}{96}+\lambda\right) Q_{1}\right], \\
& Q_{4}=192\left[T_{2}^{*}+\left(\frac{1}{24}+\lambda\right) Q_{2}-\frac{7}{192} Q_{0}\right],
\end{aligned}
$$

and for $n \geq 5$,

$$
\begin{aligned}
Q_{n}=16 n(n-1)[ & T_{n-2}^{*}+\left(\frac{1}{8(n-1)(n-3)}+\lambda\right) Q_{n-2} \\
& \left.-\frac{1}{16(n-3)(n-4)} Q_{n-4}+\frac{3}{4 n(n-1)(n-3)(n-4)} Q_{\delta}\right],
\end{aligned}
$$

where $\delta=0$ for even $n$ and 1 for odd values of $n$. Note that the first two canonical polynomials, $Q_{0}$ and $Q_{1}$, remain undefined.

If we take $\lambda=\frac{1}{4}$ and $\phi(t)=-\frac{\cosh 1}{4}$, the exact solution of $(10)$ becomes $u(t)=$ $\cosh (2 t-1)$. For a given $n$, we compute the initial Tau approximation $u_{n}(t)$ by solving the perturbed problem:

$$
\int_{0}^{1} K(t, s) u_{n}(s) d s-\frac{1}{4} u_{n}(t)=-\frac{\cosh 1}{4}+\tau_{n} T_{n}^{*}(t) .
$$

Then, using the above procedure, we generate the $k$-th iterated Tau solutions $\hat{u}_{n, k}$ and employ the supremum norm:

$$
\|w\|=\sup \{|w(t)|: 0 \leq t \leq 1\} .
$$


TABle A. Error norms $\left\|e_{n, k}\right\|$ and Tau values

\begin{tabular}{|c|c|c|c|c|c|}
\hline$k \backslash n$ & 2 & 4 & 6 & 8 & 10 \\
\hline 1 & $3.09 \mathrm{E}-1$ & $5.65 \mathrm{E}-3$ & $4.56 \mathrm{E}-5$ & $2.01 \mathrm{E}-7$ & $5.53 \mathrm{E}-10$ \\
\hline 2 & $7.41 \mathrm{E}-2$ & $4.59 \mathrm{E}-4$ & $1.28 \mathrm{E}-6$ & $3.38 \mathrm{E}-9$ & $5.58 \mathrm{E}-12$ \\
\hline 3 & $2.87 \mathrm{E}-2$ & $1.06 \mathrm{E}-4$ & $4.97 \mathrm{E}-8$ & $1.22 \mathrm{E}-10$ & $9.37 \mathrm{E}-14$ \\
\hline 4 & $1.16 \mathrm{E}-2$ & $3.94 \mathrm{E}-5$ & $7.74 \mathrm{E}-9$ & $2.52 \mathrm{E}-11$ & $2.42 \mathrm{E}-14$ \\
\hline 5 & $4.69 \mathrm{E}-3$ & $1.58 \mathrm{E}-5$ & $3.46 \mathrm{E}-9$ & $9.60 \mathrm{E}-12$ & $1.13 \mathrm{E}-14$ \\
\hline 6 & $1.90 \mathrm{E}-3$ & $6.40 \mathrm{E}-6$ & $1.43 \mathrm{E}-9$ & $3.87 \mathrm{E}-12$ & $3.77 \mathrm{E}-15$ \\
\hline 7 & $7.70 \mathrm{E}-4$ & $2.59 \mathrm{E}-6$ & $5.81 \mathrm{E}-10$ & $1.57 \mathrm{E}-12$ & $2.44 \mathrm{E}-15$ \\
\hline 8 & $3.12 \mathrm{E}-4$ & $1.05 \mathrm{E}-6$ & $2.35 \mathrm{E}-10$ & $6.35 \mathrm{E}-13$ & $8.88 \mathrm{E}-16$ \\
\hline 9 & $1.26 \mathrm{E}-4$ & $4.26 \mathrm{E}-7$ & & & \\
\hline 10 & $5.12 \mathrm{E}-5$ & $1.72 \mathrm{E}-7$ & & & \\
\hline 11 & $2.07 \mathrm{E}-5$ & $6.99 \mathrm{E}-8$ & & & \\
\hline 12 & $8.42 \mathrm{E}-6$ & $2.84 \mathrm{E}-8$ & & & \\
\hline 13 & $3.41 \mathrm{E}-6$ & $1.15 \mathrm{E}-8$ & & & \\
\hline 14 & $1.38 \mathrm{E}-6$ & $4.66 \mathrm{E}-9$ & & & \\
\hline 15 & $5.60 \mathrm{E}-7$ & $1.89 \mathrm{E}-9$ & & & \\
\hline 16 & $2.27 \mathrm{E}-7$ & $7.65 \mathrm{E}-10$ & & & \\
\hline 17 & $9.20 \mathrm{E}-8$ & $3.10 \mathrm{E}-10$ & & & \\
\hline 18 & $3.73 \mathrm{E}-8$ & & & & \\
\hline 19 & $1.51 \mathrm{E}-8$ & & & & \\
\hline 20 & $6.13 \mathrm{E}-9$ & & & & \\
\hline$\tau_{n}$ & $7.715 \mathrm{E}-2$ & $1.41 \mathrm{E}-3$ & $1.14 \mathrm{E}-5$ & $5.02 \mathrm{E}-8$ & $1.38 \mathrm{E}-10$ \\
\hline
\end{tabular}

Numerical results for approximations of order $n=2,4,6,8,10$ with various iterations $k$ are presented in Table $\mathrm{A}$, where the error norms $\left\|e_{n, k}\right\|$ are given. We note, for example, that an error of order $O\left(10^{-8}\right)$ is obtained through Tau approximations $u_{2}, u_{4}, u_{6}$ and $u_{8}$ after $17,11,3$ and 2 iterations, respectively.

TABLE B. Error norms $\left\|e_{n, k}\right\|$ and Tau values

\begin{tabular}{|c|l|l|l|l|c|}
\hline$k \backslash n$ & \multicolumn{1}{|c|}{1} & \multicolumn{1}{|c|}{2} & 3 & 4 & 5 \\
\hline & & & & & \\
0 & 1.00 & $1.33 \mathrm{E}-1$ & $1.03 \mathrm{E}-2$ & $5.48 \mathrm{E}-4$ & $2.84 \mathrm{E}-5$ \\
1 & $3.86 \mathrm{E}-1$ & $6.04 \mathrm{E}-2$ & $2.72 \mathrm{E}-3$ & $9.67 \mathrm{E}-5$ & $3.57 \mathrm{E}-6$ \\
2 & $2.83 \mathrm{E}-1$ & $2.24 \mathrm{E}-2$ & $1.47 \mathrm{E}-3$ & $3.78 \mathrm{E}-5$ & $1.14 \mathrm{E}-6$ \\
3 & $1.15 \mathrm{E}-1$ & $3.94 \mathrm{E}-3$ & $5.83 \mathrm{E}-4$ & $1.95 \mathrm{E}-5$ & $4.96 \mathrm{E}-7$ \\
4 & $3.17 \mathrm{E}-2$ & $2.37 \mathrm{E}-4$ & $1.40 \mathrm{E}-4$ & $6.49 \mathrm{E}-6$ & $1.71 \mathrm{E}-7$ \\
5 & $6.72 \mathrm{E}-3$ & $7.94 \mathrm{E}-5$ & $2.43 \mathrm{E}-5$ & $1.49 \mathrm{E}-6$ & $4.52 \mathrm{E}-8$ \\
6 & $1.16 \mathrm{E}-3$ & $2.79 \mathrm{E}-5$ & $3.28 \mathrm{E}-6$ & $2.62 \mathrm{E}-7$ & $9.20 \mathrm{E}-9$ \\
7 & $1.70 \mathrm{E}-4$ & $5.81 \mathrm{E}-6$ & $3.52 \mathrm{E}-7$ & $3.72 \mathrm{E}-8$ & $1.50 \mathrm{E}-9$ \\
8 & $2.17 \mathrm{E}-5$ & $9.15 \mathrm{E}-7$ & $2.97 \mathrm{E}-8$ & $4.44 \mathrm{E}-9$ & $2.04 \mathrm{E}-10$ \\
9 & $2.45 \mathrm{E}-6$ & $1.19 \mathrm{E}-7$ & $1.82 \mathrm{E}-9$ & $4.57 \mathrm{E}-10$ & $2.37 \mathrm{E}-11$ \\
10 & $2.48 \mathrm{E}-7$ & $1.34 \mathrm{E}-8$ & $4.35 \mathrm{E}-11$ & $4.14 \mathrm{E}-11$ & $2.42 \mathrm{E}-12$ \\
11 & $2.28 \mathrm{E}-8$ & $1.33 \mathrm{E}-9$ & $7.61 \mathrm{E}-12$ & $3.33 \mathrm{E}-12$ & $2.19 \mathrm{E}-13$ \\
12 & $1.91 \mathrm{E}-9$ & $1.19 \mathrm{E}-10$ & $1.52 \mathrm{E}-12$ & $2.34 \mathrm{E}-13$ & $1.78 \mathrm{E}-14$ \\
& & & & & \\
\hline \hline$\tau_{n}$ & 1.00 & $1.11 \mathrm{E}-1$ & $8.85 \mathrm{E}-3$ & $5.48 \mathrm{E}-4$ & $2.72 \mathrm{E}-5$ \\
\hline
\end{tabular}


Example B. Our next example is concerned with the exponential function $e^{t}$ which satisfies the following Volterra equation of the second kind

$$
(F u)(t)=\int_{0}^{t} u(s) d s-u(t)=-1, \quad t \in[0,1] .
$$

The perturbed equation becomes

$$
\int_{0}^{t} u_{n}(s) d s-u_{n}(t)=-1+\tau_{n} T_{n}^{*}(t)
$$

and we list the numerical results in Table B.

\section{ADDENDUM}

Here we prove the following result:

Let $H$ be a separable Hilbert space with an orthonormal basis $\left\{x_{k}: k \geq 0\right\}$ and let $\left\{H_{n}: n \geq 0\right\}$ be a sequence of subspaces of $H$ such that $H_{n}^{\perp} \subset H_{n-1}^{\perp}$ for all $n \geq 1$. Then we have

$$
\operatorname{diam}\left(H_{n}^{\perp}\right) \rightarrow 0 \quad \text { as } n \rightarrow \infty,
$$

where $\operatorname{diam}\left(H_{n}^{\perp}\right)=\sup \left\{\|a-b\|: a, b \in H_{n}^{\perp}\right\}$.

To prove this, let $a=\sum_{i \geq 0}\left\langle a, x_{i}\right\rangle x_{i}$ and $b=\sum_{i \geq 0}\left\langle b, x_{i}\right\rangle x_{i}$ be two elements of $H_{n}^{\perp}$. Then

$$
a-b=\sum_{i \geq 0}\left(\left\langle a, x_{i}\right\rangle-\left\langle b, x_{i}\right\rangle\right) x_{i}=\sum_{i \geq 0}\left\langle a-b, x_{i}\right\rangle x_{i}=\sum_{i>n}\left\langle a-b, x_{i}\right\rangle x_{i} .
$$

Since the set $\left\{x_{k}: k \geq 0\right\}$ forms an orthonormal basis, we get

$$
\|a-b\|^{2}=\sum_{i>n}\left|\left\langle a-b, x_{i}\right\rangle\right|^{2} .
$$

But

$$
\sum_{i \geq 0}\left|\left\langle a-b, x_{i}\right\rangle\right|^{2}<\infty .
$$

Thus (14) is the residual of the absolutely convergent series (15) and therefore

$$
\lim _{n \rightarrow \infty} \sum_{i>n}\left|\left\langle a-b, x_{i}\right\rangle\right|^{2}=0
$$

from which it follows that $\operatorname{diam}\left(H_{n}^{\perp}\right) \rightarrow 0$.

\section{REFERENCES}

1. M. Cotlar and R. Cignoli, An Introduction to Functional Analysis. North-Holland, Amsterdam, 1974. MR 53:8845

2. L.V. Kantorovich and G.P. Akilov, Functional Analysis, Pergamon Press, Oxford, second edition, 1982. MR 83h:46002

3. E.L. Ortiz, The Tau Method, SIAM J. Numer. Anal., 6:480-492, 1969. MR 41:2934

4. Canonical polynomials in the Lanczos' Tau Method, Studies in Numerical Analysis

(B.K.P. Scaife, editor), pages 73-93. Academic Press, New York, 1974. MR 57:14478

Applied Sciences Department, College of Technical Studies, Paaet, P. O. Box 42325, SHUWAIKH 70654, KUWAIT

E-mail address: hkhajah@kuc01.kuniv.edu.kw 